\title{
Integrated Joint Actuator for Serpentine Robots
}

\author{
Grzegorz Granosik and Johann Borenstein, Member, IEEE
}

\begin{abstract}
Serpentine robots, also sometimes called "snake robots," are slender, multi-segmented vehicles designed to provide greater mobility than conventional wheeled or tracked robots. Serpentine robots typically comprise of three or more rigid segments that are connected by 2- or 3-degrees-of-freedom (DOF) joints. The segments typically have powered wheels, tracks, or legs to propel the vehicle forward, while the joints may be powered or unpowered.

We have developed a joint actuator system that is highly optimized for use in serpentine robots. This paper first presents an analysis of the particular requirements for joint actuators in serpentine robots. We then compare existing actuators against those requirements and show that pneumatic bellows are ideally suited for this application.

Following this analysis the paper introduces our fully functional, pneumatically operated actuation system that is efficiently integrated in the space occupied by a joint. This system, which we call "Integrated Joint Actuator," also allows simultaneous proportional control of position and stiffness of the joint. The key advantages of our design over other joint actuation methods are its great strength combined with controllable compliance and minimal space requirements.
\end{abstract}

Index Terms - serpentine robots, pneumatics, position and stiffness control

\section{INTRODUCTION AND BACKGROUND}

Many mechanical systems exist, in which two members are linked by a joint that allows one, two, or more Degrees-ofFreedom (DOF) of motion between the members. Application areas for such systems are robotics in general and, more specifically, so-called "snake" or "serpentine" mobile robots. Such serpentine robots typically comprise three or more rigid segments and joints connecting those segments. Because of ambiguity in the use of the terms "snake robot" and "serpentine robot," we introduce the following definition for the remainder of this paper.

- A "snake robot" or (snake-like robot) is a multi-segment mechanism that derives propulsion from the relative motion of the joints only, that is, it uses no wheels, legs, or tracks for propulsion.

- A "serpentine robot" is a multi-segment mechanism that

Manuscript received November 16, 2003; revised July 8, 2004. This work was conducted at the University of Michigan's Mobile Robotics Lab and funded by the U.S. Department of Energy under Award No. DE-FG0486NE3796.

G. Granosik is with Technical University of Lodz, Institute of Automatic Control, Lodz, POLAND (phone: +4842 631 2554, fax: +4842 631 2551, e-mail: granosik@p.lodz.pl).

J. Borenstein is with The University of Michigan, Dept. of Mechanical Engineering, Ann Arbor, MI, USA (e-mail: johannb@umich.edu) derives propulsion from wheels, legs, or tracks. Joints connecting the segments may be either powered or unpowered.

\section{A. Review of existing snake and serpentine robots}

Snake-like robots have been attracting the attention of researchers since the seventies. Around that time Shigeo Hirose from the Tokyo Institute of Technology developed his Active Cord Mechanism, which mimicked snake movements. The latest incarnation of this idea is the ACM-R3 robot, which is capable of performing new types of three-dimensional snake-like locomotion [Mori and Hirose, 2002].

In the nineties research on snake-like robots increased dramatically as documented by Dowling [1997]. Muth and Grant [2000] developed the MOCASIN II pipe crawler. This snake-like robot uses joint actuators for active propulsion while actuators embedded in the segments are used for holding consecutive links in place. Another snake-like robot that uses pneumatic power for actuating its joints is the Slime Robot (SR) developed by Ohno and Hirose [2000]. Metal bellows used in the initial prototype were changed to bridle bellows in the latest version, called SSR-II [Aoki et al. 2002].

Fewer examples exist in the category of serpentine robots, and they are all the result of more recent projects. The first practical realization of a serpentine robot, called KR-I, was introduced by Hirose and Morishima [1990] and the improved version KR-II was presented by Hirose et al. [1991]. More recently, Klaassen and Paap [1999] at the GMD developed the Snake2 vehicle, which contains six active segments and a head. Each round segment has an array of 12 electrically driven wheels evenly spaced around its periphery. These wheels provide propulsion regardless of the vehicles orientation (i.e., its roll angle). Segments are interconnected by universal joints actuated by three additional electric motors through strings. Another serpentine robot designed for sewer inspection was developed by Scholl et al. [2000]. Its segments use only two wheels but the actuated 3-DOF joints allow full control over each segment's spatial orientation.

While wheeled serpentine robots can work well in smoothwalled pipes, more rugged terrain requires tracked propulsion. To this effect Takayama and Hirose [2000] developed the Soruyu-I crawler, which consists of three segments. Each segment is driven by a pair of tracks, which, in turn, are all powered simultaneously by a single motor, located in the center segment. Each distal segment is connected to the center segment by a special 2-DOF joint mechanism, which is actuated by two lead screws driven by two electric motors.

A different concept using unpowered joints was introduced by Kimura and Hirose [2002]. That robot, called Genbu, is 
probably the only serpentine robot with unpowered joints. The stability of the robot and its high mobility on rough terrain are preserved by large-diameter wheels $(220 \mathrm{~mm})$.

A serpentine robot that is strikingly similar to our OmniTread serpentine robot (introduced in Section B) is MOIRA [Osuka and Kitajima, 2003] ${ }^{1}$. MOIRA comprises four segments, and each segment has two longitudinal tracks on each of its four sides, for a total of eight tracks per segment. The 2-DOF joints between segments are actuated by pneumatic cylinders. We believe that the bellows-based joint actuators used in our OmniTread have a substantial advantage over a cylinder-based design, as the discussion of our approach in Section $\mathrm{V}$ will show.

The concept of joining several small robots into a train to overcome larger obstacles was used by Brown et al. [2002] in their Millibot Train. The robot has been demonstrated to climb up a regular staircase and even higher steps. However, with only one DOF in each joint the vehicle is kinematically limited.

\section{B. University of Michigan-developed serpentine robots}

Our own two serpentine robot designs, called "OmniPede" and "OmniTread," are shown in Figures 1 and 2, respectively. Insights gained from the earlier work on OmniPede helped us design the OmniTread. This tracked robot is about 5-10 times more energy efficient than the legged OmniPede.

One key problem with the OmniPede and other serpentine robots is best explained by introducing an artificial, dimensionless unit that we call "Propulsion Ratio" $P_{r}$. $P_{r}$ is measured as the surface area that provides propulsion, $A_{p}$, divided by the total surface area of the body

$$
P_{r}=A_{p} /\left(A_{p}+A_{i}\right)
$$

where $A_{i}$ is the inert (non-propelling) surface area of the body. The propulsion ratio for the OmniTread is about $P_{r}=0.6$, whereas that ratio for the OmniPede is almost one order of magnitude smaller. One other important fact related to propulsion ratio is that the space taken up by the joints, which we call "Joint Space," should be as small as possible because it typically has only inert surface areas that reduce $P_{r}$.

The advantages discussed above make the OmniTread far more practical than the OmniPede, and we will refer only to the OmniTread in the remainder of this paper.

The dominant features of all serpentine robots are their elongated structure and multi-segmented construction. These features require two drive mechanisms, one for propulsion and one for joint actuation. In our Omni-robots we addressed this problem by using a single motor that provides torque to all propulsion elements via a so-called drive shaft spine (see Long et al. [2002]). While we believe that this design is the most space- and weight-efficient approach, the transmission mechanism in each segment still occupies about $1 / 3$ of the

\footnotetext{
${ }^{1}$ Osuka and Kitajimas effort and ours are independent. We became aware of their work through their presentation/publication in October 2003. However, the development of our two serpentine robots, OmniPede and OmniTread, began in 1998 and September 2002, respectively.
}

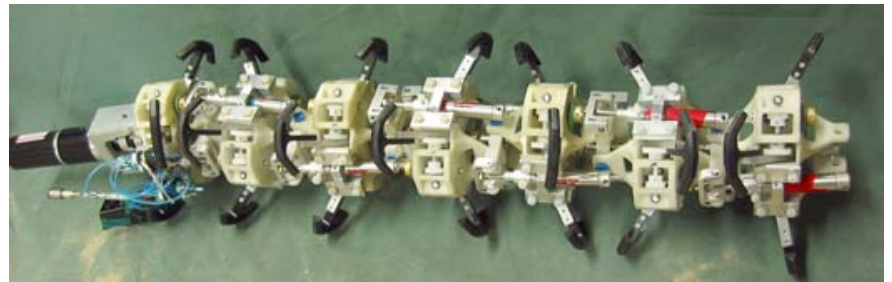

Figure 1: The "OmniPede," developed at our lab, is a 7-segment serpentine robot that uses legs for propulsion. An electric motor at the end rotates a socalled "drive shaft spine," which provides mechanical power to each foot (black parts) through a five-bar mechanism. Segments are linked by 2-DOF articulate joints that are actuated by two pneumatic cylinders.

internal space of the segment.

Some additional space can be found between segments, besides the two-degrees-of-freedom (2-DOF) articulate joints. With space being a key limitation in serpentine robots, it is important that this so-called "Joint Space” be used efficiently. This, along with the other functional requirement listed in Section C, motivated the design of our integrated joint actuators.

\section{Requirements for joint actuation in serpentine robots}

By definition, serpentine robots are relatively long compared to their diameter, so that their lead segments can reach up and over a high obstacle while still being able to fit through small openings. From this geometric constraint, as well as from other unique operational characteristics of serpentine robots, the following requirements for joint actuators can be derived:

1. The energy consumption and weight of the actuators should be minimal, because energy is a limited resource in an untethered mobile robot.

2. Serpentine robots should conform to the terrain compliantly, so that as many driving segments as possible are in contact with the ground at all times to provide effective propulsion.

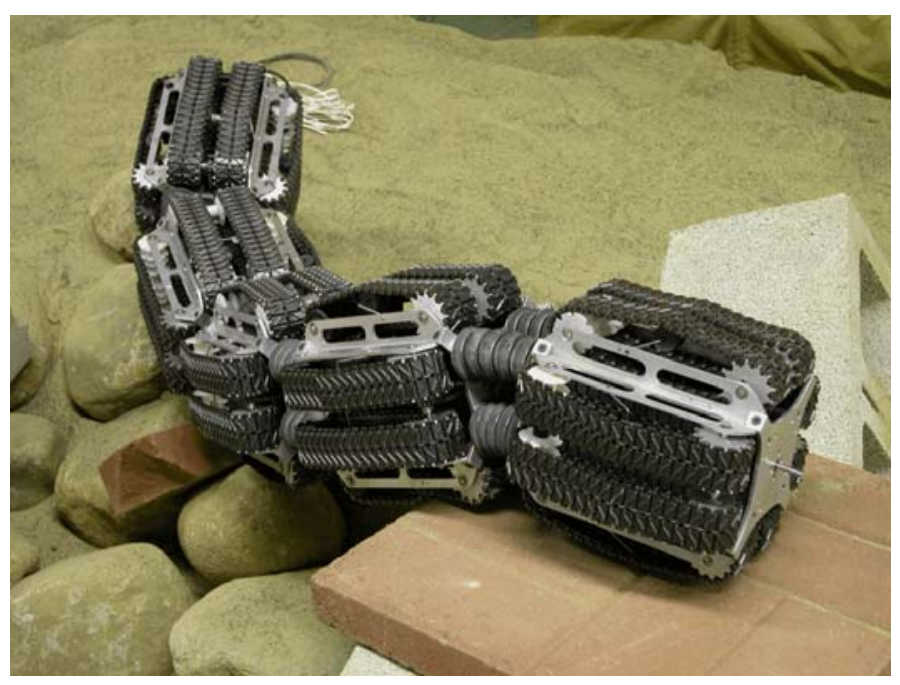

Figure 2: The OmniTread serpentine robot developed at our lab. As in the OmniPede, a single drive motor housed in the center segment powers a drive shaft spine, from which torque is derived for all treads. All segments are linked by 2-DOF pneumatically actuated joints, which are the focus of this paper. 
3. At other times it is necessary to increase the stiffness of a joint, for example, for crossing a gap or reaching over an obstacle. Serpentine robots must thus be capable of adjusting the stiffness of every degree of freedom individually and proportionally.

4. Joint angles in serpentine robots should be controllable proportionally, to provide full 3D mobility.

5. Joint actuators should be capable of developing sufficient force to lift at least two lead segments to the edge of a step, in order to climb over it.

6. As discussed in Section B, large amounts of space dedicated to joints dramatically increase the amount of inert surface area and limit mobility on rugged terrain. Therefore joint actuators should take up as little space as possible, to reduce the size of joint space.

To meet the unique set of requirements for joint actuators in serpentine robots, we took a methodical approach, which is followed, in essence, by the layout of this paper. In Section 2 we review possible joint actuators and discuss their strengths and weaknesses. Section 3 discusses different pneumatic joint actuators, and Section 4 provides a practical solution for the proportional control of the position and stiffness of pneumatic actuators. Section 5 presents our design of an integrated joint actuator for serpentine robots, which meets all of the requirements listed above. Section 6 presents experimental results and Section 7 offers our conclusions.

\section{REview of CANDidATE Joint Actuators}

There are many different ways of actuating joints in a mechanical structure. However, only a few of them can provide the range of motion and force required for actuating the joints of a serpentine robot. Those actuators are electrical motors, hydraulic motors or actuators, and pneumatic actuators. Table I lists some key parameters for candidate joint actuators.

\section{A. Actuation Stress/Strain analysis}

In order to find the best-suited actuator for joints in serpentine robots we performed a detailed analysis mostly based on the comparison of performance indices of

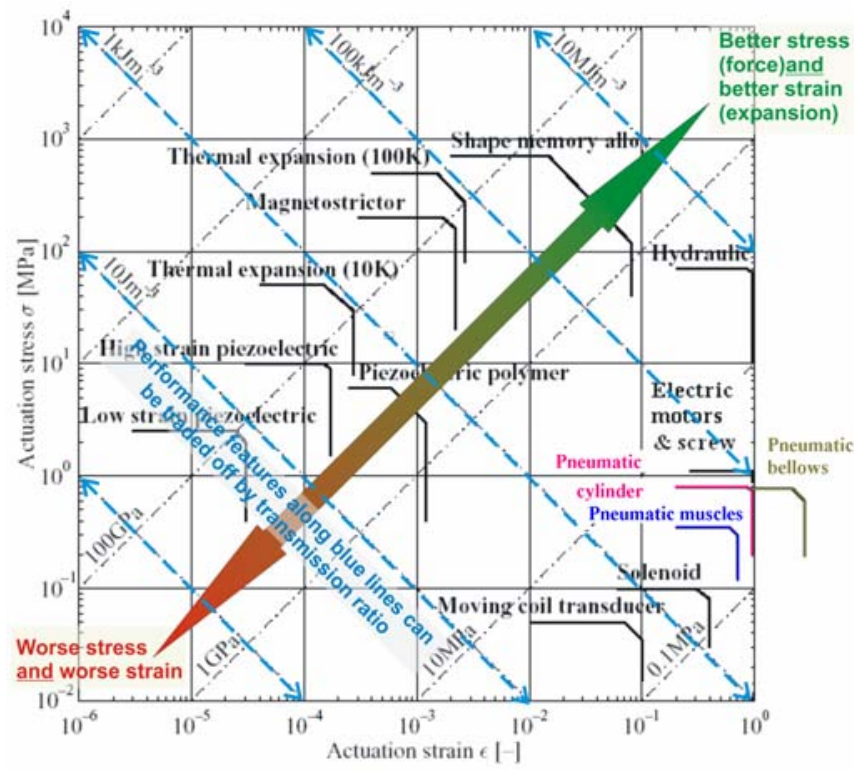

Figure 3: Actuation stress versus actuation strain for various actuators (reproduced from Huber et al. [1997] and augmented with own data.)

mechanical actuators introduced by Huber et al. [1997] and complemented by our own investigations.

The original paper did not include electric motors. It also included only select types of pneumatic actuators. We made some reasonable assumptions about the transformation of rotary motion to linear motion and calculated the performance indices for some electric motors with a ball screw transmission mechanism that produces reasonable linear speed and force. We also calculated the performance indices for a few pneumatic bellows and artificial pneumatic muscles and added those results in Figure 3.

Actuators that are closest to the top right corner of Figure 3 are naturally suited to lifting weights and propelling masses in the orders of magnitude required for serpentine robots.

The superior characteristics of hydraulics (compared to pneumatics) are diminished once actuation stress is related to actuator density. Furthermore, hydraulics also becomes less desirable over electric motors once efficiency is considered, as was shown in Table I. One should also note that Huber's

TABLE I: KEY PARAMETERS OF DIFFERENT ACTUATORS (REPRODUCED FROM [OLSZEWSKI, 1998])

\begin{tabular}{|l|c|c|c|}
\hline Drive type performance compared & Electric & Hydraulic & Pneumatic \\
\hline Efficiency* [\%] & $(<1) 50-55(>90)$ & $30-35$ & $15-25$ \\
\hline Power to weight ratio [W/kg] & $25-150$ & 650 & 2000 \\
\hline Force to cross section area [N/cm2] & $0.3-1.5$ & $6 \cdot 10^{6}$ & 300 \\
\hline Durability [cycles] & $5-9 \cdot 10^{5}$ & 30 & $>100$ \\
\hline Stiffness [kN/mm] & $10-120$ & 50 & 1 \\
\hline Overload ratio [\%] & 25 & $0.02-2$ & $0.002-2$ \\
\hline Linear movements ranges [m] & $0.3-5$ & $0.1-0.05$ & $0.05-3$ \\
\hline Linear velocity [m/s] & $0.001-5$ & Worse & $0.05-30$ \\
\hline Positioning precision [mm] & 0.005 & Higher & 0.1 \\
\hline Reliability (relative) & Normal & Better \\
\hline Maintenance costs (relative) & Normal & Lower \\
\hline Unfavorable features & Electric hazard, magnetic \\
disturbances, heating & Leakages, difficulties with \\
energy transmission
\end{tabular}

Note: The efficiency value in this table already includes a "penalty" for producing pneumatic or hydraulic pressure from a rotary source of mechanical power. 
analysis considers the actuator only, but without the volume (or weight) of the compressor, manifolds, valves, fittings, and pipes. It is difficult to calculate the performance indices for the whole actuation system with precision because the volume and weight strongly depends on the application. In general these weight factors work in favor of electric systems. Once these considerations are taken into account, in addition to the actuation stress/strain analysis illustrated by Figure 3, it appears evident that there is some advantage to electric motors. However, the fact remains that actuation strain of most cylinder-type actuators is limited to 1.0 and only pneumatic bellows produce the largest value (reaching 4) without any external mechanisms. Also overload ratio of pneumatic actuators is significantly higher then competitors.

\section{B. Natural Compliance}

The actuation stress/strain analysis and discussion in the proceeding section showed some apparent advantage for electric motors, with respect to the actuation of joints in serpentine robots. However, there is another consideration, which, in our opinion, is of primary importance: natural compliance. We believe that natural compliance is critical for robots, whose propulsion depends on optimal traction between its propulsion elements (i.e., legs, wheels, or treads) and arbitrarily shaped environments, such as the rubble of a collapsed building or the rugged floor of a cave.

As can be find in Huber's work, the lines of slope +1 in Figure 3 are related to the stiffness of the actuators. Hydraulic systems provide several orders of magnitude greater stiffness than pneumatic systems, which, in turn, are stiffer then electric motors without closed loop position control. But electric motors do require closed-loop control and have to be considered in this configuration. That means that the working stiffness of electric motors depends on parameters of the control loop. However, this is true for the motors only; if gearboxes or transmissions are added, then elasticity is eliminated. This makes electric drives ideal for accurate position control, but not for compliance. Serpentine robots that don't use compliant actuators require extremely complex sensor systems that measure contact forces and command a momentary angle for each non-compliant joint so as to force contact with the ground. Such actively controlled compliance has not yet been successfully demonstrated, and may well be unfeasible for many more years.

Robinson [2000] offered a work-around for this inherent limitation. He demonstrated that elasticity could be added to an inherently stiff actuator to allow accurate force and position-force control. He accomplished this by adding a soft spring in series with an electric motor with ball screw transmission or to a hydraulic cylinder. Special control algorithms allowed his system to produce a controllable force. However, this approach substantially reduces the actuation strain and increases the weight of the actuator, which is then no longer suitable for serpentine robots.

We therefore conclude that pneumatic actuators are the only devices that provide natural compliance. The price we pay for natural compliance is the need for onboard compressed air as well as the lower energy efficiency of the pneumatic system.

The pneumatic actuator family is located very close to electric actuators in Figure 3. In practice pneumatic actuators behave as natural air springs, and, when used in closed-loop systems, can work as position-force actuators. Moreover, changes in working pressure can control the stiffness of pneumatic actuators from very limp (compliant) to very stiff. It is this fundamentally important property that makes pneumatic actuation the preferred choice for serpentine robots.

\section{PNEUMATIC ACTUATORS}

There are three "mainstream” types of pneumatic actuators: cylinders, bellows, and artificial pneumatic muscles. Cylinders and bellows develop force in quadratic proportion to their diameter $d$. In pneumatic muscles force is related to diameter and length, and the actuation force can be much larger than the force generated by a cylinder with the same diameter. However, a larger force requires greater length of the muscle, and the force drops very quickly with contraction. The actuation force of bellows also drops with expansion, but not nearly as dramatically as that of muscles. The pneumatic bellows developed at the University of Michigan with their static characteristics are shown in Figure 4.

The designers of the serpentine robot MOIRA [Osuka and

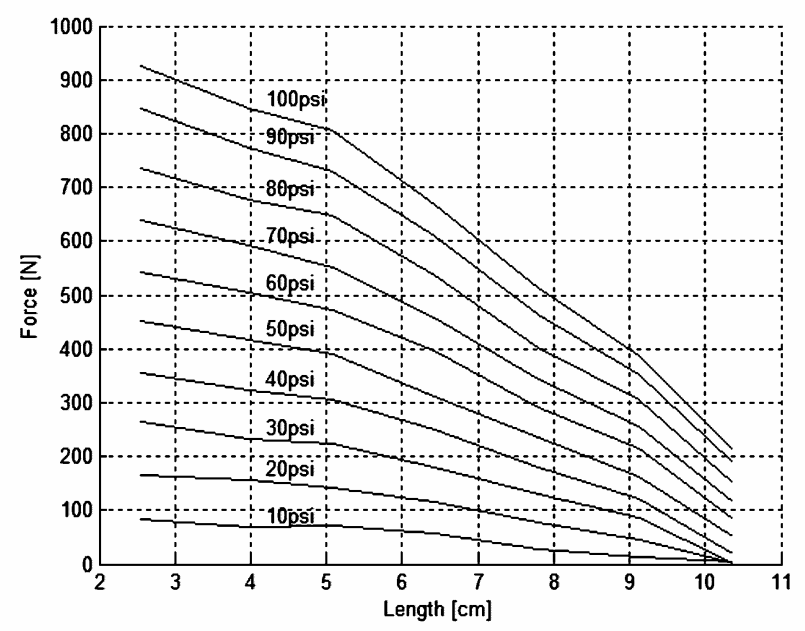

a. Static characteristics of pneumatic bellows

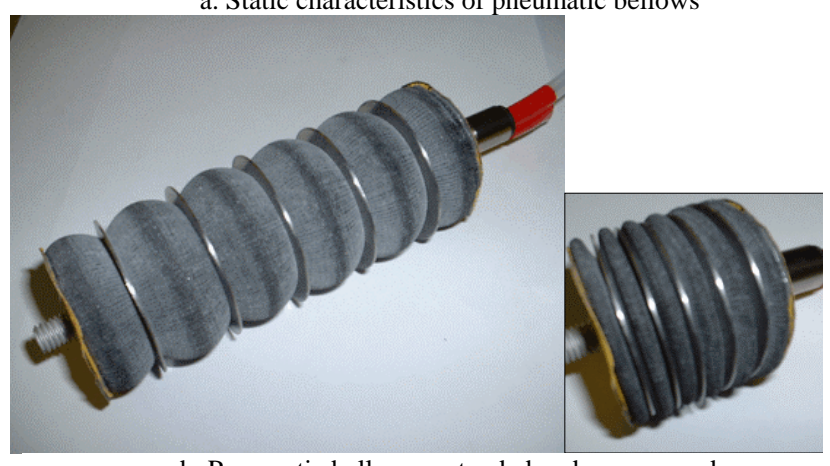

b. Pneumatic bellows, extended and compressed Figure 4: Pneumatic bellows developed at University of Michigan. Kitajima, 2003] chose to place the cylinder-type pneumatic actuators in the space of the joints. As a result, joints take up 
even more space than segments. We believe that this is a less advantageous design, because doing so increases the robot's inert surface area $A_{i}$ and thus reduces the propulsion ratio $P_{r}$, discussed in Section I.B.

To avoid this situation, cylinders or pneumatic muscles would have to be placed within a segment to actuate the joints. These actuators would take up much or most of the available space within a segment. This, in turn, would dramatically limit the space available for the mechanical drive components, pneumatic valves, and electronic components.

In contrast to cylinders and artificial muscles, pneumatic bellows are an ideal solution, because they allow the integration of one or more large-diameter pneumatic actuators in the space of the joint, without requiring any space within a segment. As shown in Figure 6, in contrast to rigid components, bellows have the very suitable property of taking up minimal space when deflated, and maximal space when inflated. They can thus be placed in joint space, without taking up any segment space. This property of bellows is highly desirable because of the severely limited space in a serpentine robot.

Furthermore, the location of the pneumatic actuators in joint space allows for larger actuator diameters than what would be possible if the actuators had to be placed in segment space, where space is shared with all other onboard components.

\section{PROPORTIONAL POSITION AND STIFFNESS CONTROL}

So far we have shown that pneumatic bellows are a good, or even the best solution, for meeting requirements \#1, \#2, \#5, and \#6 in Section I.C. We will now present a solution for meeting requirements \#3, \#4. These requirements, which call for the proportional control of position and stiffness of the joints, are not easy to meet with pneumatic actuators.

Traditionally pneumatic systems were designed for socalled pick and place operations, which is obviously too limited for the actuation of joints in serpentine robots.

More advanced methods, which allow the proportional

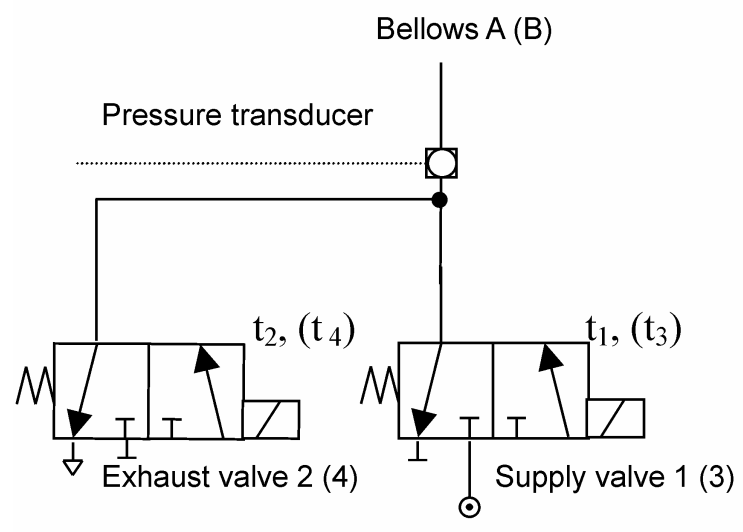

Figure 5: Pneumatic circuit for our Proportional Position and Stiffness (PPS) controller with zero air consumption at steady state. The location of Bellows $\mathrm{A}$ and $\mathrm{B}$ is shown in Figure 6. control of pneumatic actuators, were introduced in recent years [Van Varseveld and Bone, 1997; Shih and Ma, 1998]. Common to these proportional control methods is their continuing consumption of compressed air, both during motion and while holding still. This is not a problem in conventional (i.e., industrial) pneumatic systems where there is usually a local source of compressed air that can provide an almost unlimited supply of compressed air at little cost. For mobile robots requiring pneumatic actuation, however, these proportional control methods are not suitable because of their continuous consumption of compressed air.

Another approach to implementing pneumatic proportional control is based on the use of servo valves. While pneumatic servo valves can be very precise, they also tend to be heavy and bulky. They are thus more suitable for stationary manipulators [Bobrow and McDonell, 1998] and less so for mobile robots.

For smaller mobile robots much lighter and compact on-off valves are a more applicable solution. Moreover, reduction in air consumption was achieved by a four-valve configuration proposed by Galt et al. [1997] and refined by Brockmann and Köhne [2001]. In their method the chambers of cylinder-type actuators were closed in steady state and thereby preserved compressed air. However, in this work the stiffness of the joints was not controlled. In serpentine robots and certain other applications stiffness must be controlled at all times. For example, when multiple segments of a serpentine robot span a gap, maximum stiffness must be maintained. In contrast, when traveling across rugged terrain minimal stiffness (=maximal compliance) must be maintained. Alternatively, it may be necessary to adjust the stiffness to an intermediate level, for example, when the lead segment leans against a vertical wall while being pushed up that wall by the following segments.

In order to provide proportional position control and proportional stiffness control simultaneously, as well as zeroairflow at steady state, we developed what we call the "Proportional Position and Stiffness (PPS)" controller. Figure 6 shows a simplified 1-DOF joint operated by two bellows and controlled by the pneumatic circuit shown in Figure 5. Figure 7 shows the control block diagram for the PPS controller.

The PPS control algorithm is based on the simplified dynamic model of a bellows-driven joint, given by

$$
I \ddot{q}=\tau_{p}-\tau_{s}-\tau
$$

where:

$$
\begin{aligned}
& I-\text { polar moment of } \\
& \text { inertia of parts } \\
& \text { rotating around joint } \\
& \text { 'O' in Figure } 6 \text {, } \\
& q-\text { joint angle, } \\
& \tau_{s} \text { - torque around ' } \mathrm{O} \text { ' } \\
& \text { caused by elasticity } \\
& \text { of inflated bellows. }
\end{aligned}
$$
We observed that this

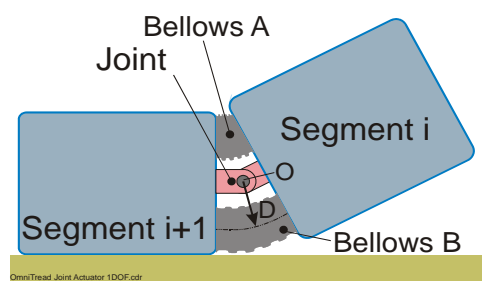

Figure 6: A simplified 1-DOF joint with bellows-type actuators. component could be neglected for our bellows with no 


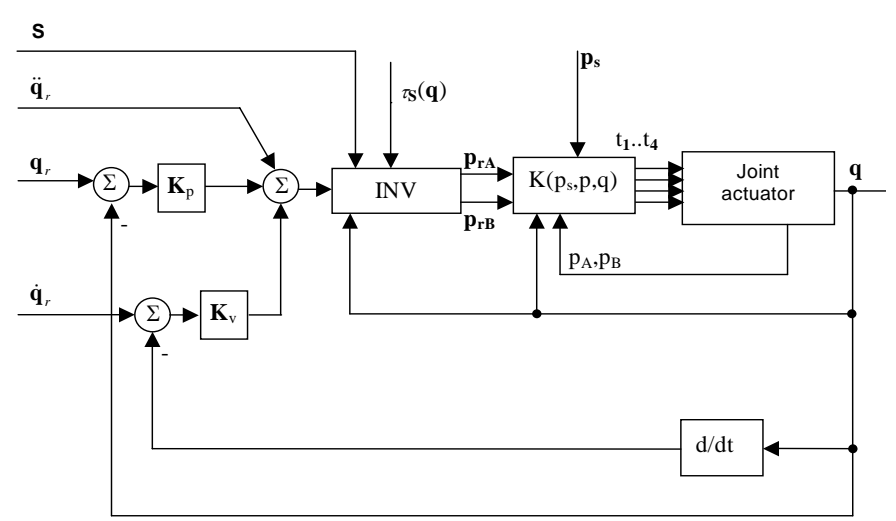

Figure 7: Block diagram of control system for our Proportional Position and Stiffness (PPS) controller with zero air consumption at steady state.

loss in the quality of trajectory following,

$\tau$ - load torque, generated by the weight of the lifted segment(s),

$\tau_{p}-$ net torque generated by Bellows $\mathrm{A}$ and $\mathrm{B}$, and given by

$$
\tau_{p}=D A\left(p_{A}-p_{B}\right)
$$

where:

$D$ - length of lever arm of torque-generating Bellows A and $\mathrm{B}$,

A - cross section area of Bellows A and B.

$p_{A}, p_{B}-$ pressures in opposite Bellows A and B in Figure 6.

The actual control algorithm employed by our PPS controller is the so-called "inverse model" [Craig 1989] and is therefore shown as block "INV" in Figure 7. The task of Block INV is to generate reference values for the pressures $p_{r A}$ and $p_{r B}$, which are then passed on to the pressure controller. Block "INV" incorporates Eq. (2), which represents the sum of all torques acting in the simplified 1-DOF joint of Figure 6. In steady state, the difference between the pressures in Bellows A and B determines the actuation moment that the bellows apply to the joint, as shown by Eq. (3). The sum of the pressures in Bellows $\mathrm{A}$ and $\mathrm{B}$, determines the angular stiffness of the joint [Liu et al. 1996; Granosik and Jezierski 1999]. Thus, both the actuation torque and the angular stiffness can be controlled simultaneously by selecting appropriate pressures $p_{A}$ and $p_{B}$.

Moreover, the proposed control system assigns higher priority to stiffness when conflicts between position control and stiffness control arise. For example, conflicts may arise when the controller tries to reach a commanded position while being commanded to maintain low stiffness $S$. In this case, both $p_{A}$ and $p_{B}$ must be small, and the pressure difference, which produces the torque for moving the joint to the commanded position, may be to small to do so. In the case of such conflicts we assigned higher priority to stiffness control than to position control.

In order to derive our control approach, we make the important assumption that the dynamics of compressed air in a pair of bellows is similar to that in the two chambers of a cylinder. Based on this assumption, we adopted the expression derived by Shearer [1956] for the latter:
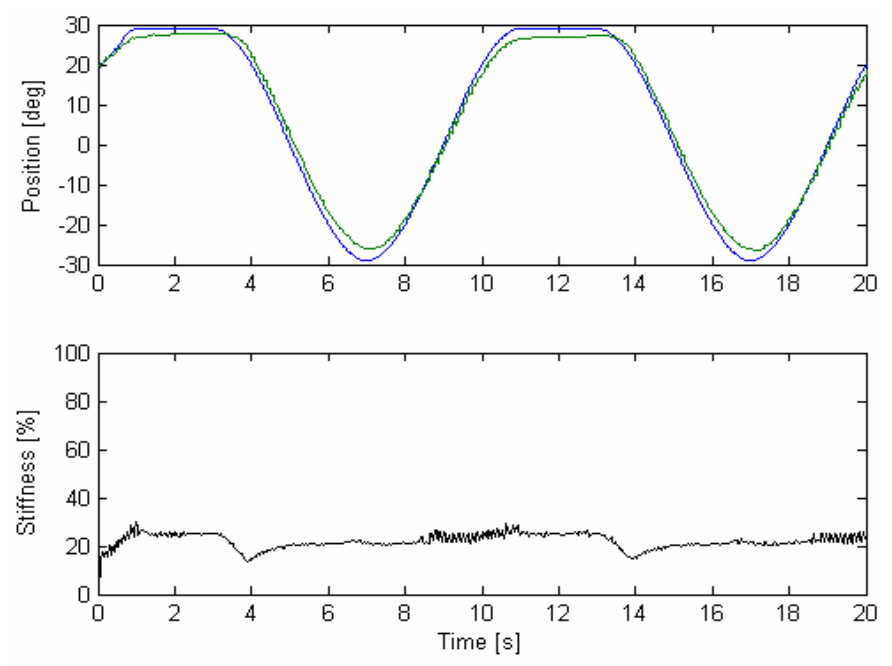

Figure 8: Experimental results with the PPS controller. Trajectory following with constant stiffness.

$$
\dot{p}=\frac{k R T \dot{m}}{V}-\frac{k p \dot{V}}{V}
$$

where:

$p, V, \dot{p}_{r}, \dot{V}$ - pressure in bellows, volume of bellows, and first derivatives of pressure and volume, respectively,

$k$ - ratio of specific heats (for air $k=1.4$ ),

$R, \dot{m}, T$ - gas constant, mass airflow and temperature of compressed gas, respectively.

Pressures $p_{A}$ and $p_{B}$ are controlled in our system by means of pulse width modulation (PWM), which is realized in block $\mathrm{K}\left(p_{s}, p, q\right)$ in Figure 7. The PWM controller functions by modifying the fraction of time $t_{i}(i=1 . .4)$, during which certain valves of Figure 5 are open (in the case of exhaust valves) or closed (in the case of supply valves) during every PWM interval $t_{p}$.

We can compute the control parameter $t_{i}$ according to (5)

$$
t_{i}=\left\{\begin{array}{lll}
G \frac{t_{p}}{A_{s} \sqrt{p_{s}-p}} & \text { for } & G \geq 0 \\
G \frac{-t_{p}}{A_{e} p} & \text { for } & G<0
\end{array}\right.
$$

using energy flow $\mathrm{G}$ derived from (4) as:

$$
G=R k T \dot{m}=K_{D P} V \dot{e}_{p}+K_{P P} V e_{p}+k p \dot{V}
$$

where:

$t_{p}$ - period of the pulse width modulation (PWM) controller,

$t_{i}$ - length of a pulse in the PWM controller. Four different signals $t_{1} . t_{4}$ control the respective valves as shown in Figure 5. $t_{i}$ is always a fraction of $t_{p}$,

$e_{p}, \dot{e}_{p} \quad$ - pressure regulation error and its first derivative, respectively,

$K_{D P}, K_{P P}$ - derivative and proportional coefficients of PD pressure regulator.

$A_{s}=0.094$ and $A_{e}=-0.099$ are experimentally determined coefficients that apply for the case of $G>0$ and $G<0$, respectively. $G$ is proportional to mass airflow and its sign describes the direction of airflow. A positive value for $G$ 


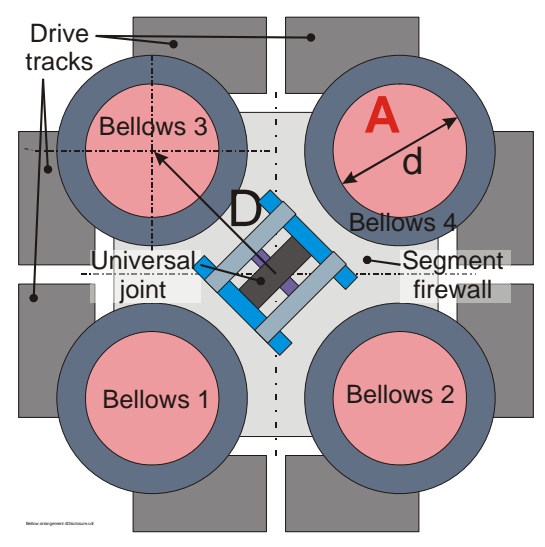

Figure 9: Cross-section of the integrated joint actuator.

indicates that air is being supplied to the bellows and that valve number 1 or 3 (see Figure 5) is closed. A negative value for $\mathrm{G}$ indicates that air is being exhausted and that valves 2 or 4 are opened.

Figure 8 shows an experiment, in which a pair of pneumatic bellows was controlled to expand and contract in a sinusoidal fashion, for two full periods. The PPS controller maintains a near-constant stiffness of $20 \%$, as was commanded in this experiment. In our controller, stiffness does not have to be constant; it can change in the full range of $0 \%-100 \%$ under computer control and in real time. Both degrees of freedom of each joint are controlled independently using our PPS controller. However, to avoid possible conflicts we command the same stiffness for the entire joint.

\section{The INTEgrated Joint ACTUATOR IN SERPENTINE ROBOTS}

Based on the discussion thus far we have chosen pneumatic bellows as the best-suited actuator for serpentine robots. In accordance with that choice we designed the "Integrated Joint Actuator” (IJA) for serpentine robots. Figure 9 shows a crosssection of the IJA. The design assumes that there is a 2-DOF universal joint in the center, connecting any two adjacent segments. An arrangement of four equally spaced bellows is used to actuate the two degrees of freedom of each joint. Each closed end of a bellows is rigidly fastened to the front or rear "firewall" of a segment. Compressed air can be pumped into

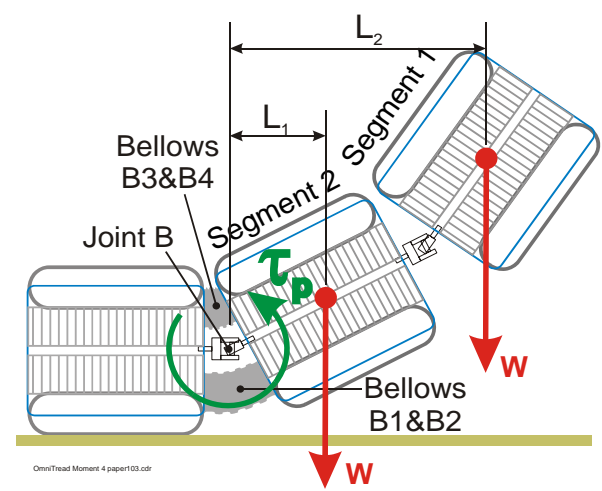

Figure 10: A typical task for a serpentine robot might involve the lifting of its first two segments to reach up to the top of a stair. the bellows or exhausted from the bellows via an appropriate hole in the firewall. The maximum bending angle in our IJA is up to $25^{\circ}$ in each direction.

In order to be able to traverse high obstacles, a serpentine robot should be able to lift as many segments as possible off the ground. As we will see below, though, the geometric shape of serpentine robots makes it extremely difficult to do so. To illustrate this problem, Figure 10 shows the case of the OmniTread lifting its two lead segments, each of weight $W$. To accomplish this task, the IJA of Joint B inflates bellows B1 and B2 and exhausts bellows A1 and A2. This creates a lifting torque $\tau_{p}$ that must overcome the reactive moment from the weight of the two segments, $M_{\text {react }}=L_{1} W+L_{2} W$.

One must further keep in mind that in a fully symmetric serpentine robot, the vehicle has no "bottom” or "top.” Rather, it can roll on any side and may even move on one of its four edges (as can be visualized by thinking of Figure 9 rotated $45^{\circ}$ clockwise or counter-clockwise). In such an extreme case, only one single bellows would be able to contribute to the lifting torque $\tau_{p}$. In this case, the lever arm for producing this lifting torque has length $D$, as shown in Figure 9.

For the worst case of the OmniTread laying on its edge, the lifting torque $\tau_{p}$ produced by a single pair of opposite bellows was given by Eq. (3). During experiments we measured the minimum value of the pressure difference $\left(p_{A}-p_{B}\right)=63$ psi needed for generating a torque $\tau_{p}=25 \mathrm{Nm}$. This torque is sufficient to lift up the two lead- or tail-segments.

In the nominal case of Figure 10 (OmniTread laying on a side, not an edge), not just one but two bellows-pairs provide the lifting torque, albeit at a reduced moment lever $D / \sqrt{2}$. The available lifting torque in that case is larger than in the case of the OmniTread laying on its edge and can be generated by an even smaller pressure difference. In this case two front segments can be lifted up by the pressure difference $\left(p_{A}-p_{B}\right)=47$ psi generating a torque $\tau_{p}=27 \mathrm{Nm}$.

\section{EXPERIMENTAL RESULTS}

Figure 11 and Figure 12 show our current (December 2003) OmniTread prototype, which comprises five segments and four joints. Each joint operated by a 2-DOF integrated joint actuator (IJA) with four bellows. The size of each segment is $20 \times 18.6 \times 18.6 \mathrm{~cm}$ (length $\times$ width $\times$ height). Each joint space

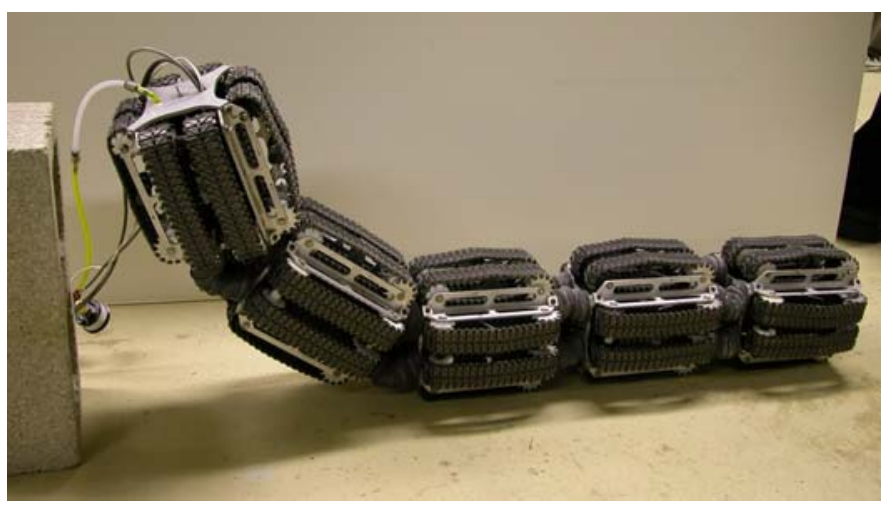

Figure 11: Experiments with OmniTread lifts up its two lead segments. 


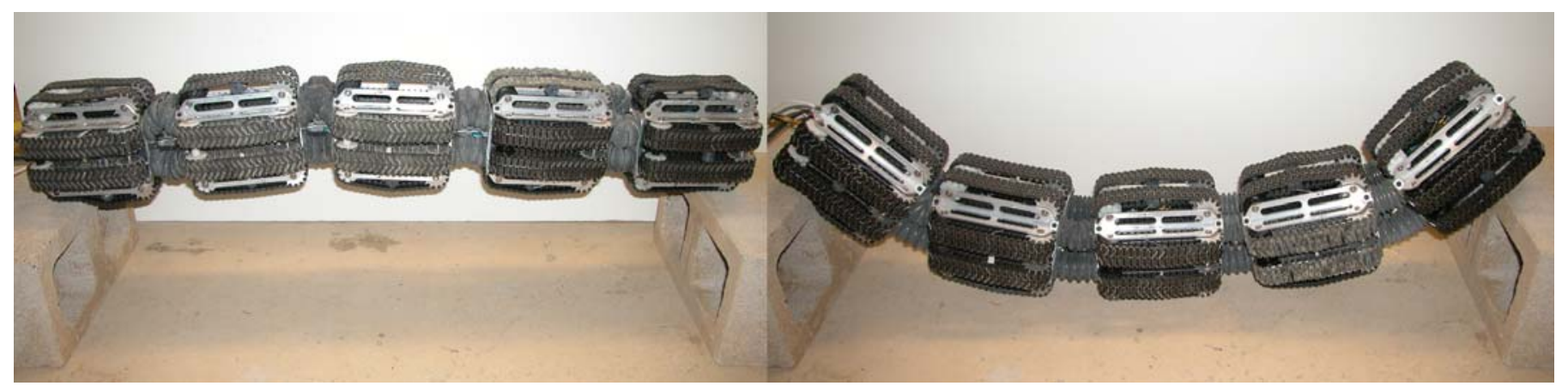

Figure 12: Experiments with active stiffness control. a) maximum stiffness - left, b) minimum stiffness - right.

We show this experiment only for illustrative purposes. In practice, our OmniTread cannot reach a situation, in which it spans a gap that is wider than half its length (assuming symmetric weight distribution). This is because the OmniTread would fall into the gap as soon as more than half of its weight is over the gap.

is $6.8 \mathrm{~cm}$ long. The entire robot is $127 \mathrm{~cm}$ long and weighs about $13.6 \mathrm{~kg}$. The minimum radius of curvature is $53 \mathrm{~cm}$.

In order to control the IJAs we developed a microprocessorbased distributed control system consisting of four local controllers - one for each IJA. Each local controller is based on a 16-bit Motorola microcontroller MC9S12DP256B and all four controllers communicate with a master PC via CAN bus. Each microcontroller realizes position and stiffness control for its local 2-DOF joint.

In our current prototype the external compressor provided variable pressure from 85 to 95 psi but the control system limited the maximum pressure in the bellows to 80 psi.

Figure 11 illustrates the IJA's ability to lift up and hold in the air the two lead segments, as would be necessary to scale an overhanging vertical obstacle. In this case the lead segment reached a height of $39 \mathrm{~cm}$ or $30.7 \%$ of total vehicle length. Figure 12a shows the advantages of active stiffness control of the joints. With stiffness set to maximum, the OmniTread can span a 108-cm gap created by two concrete blocks. When stiffness is decreased, the OmniTread conforms to the gap compliantly, as shown in Figure 12b.

\section{CONCLUSIONS}

This paper focuses on the problem of joint actuation in socalled serpentine robots. Based on our experience with the design of such robots, we defined in this paper the unique requirements for joint actuation in serpentine robots. One particularly important requirement, defined in this paper for the first time, is the need for a large "propulsion ratio." The propulsion ratio is the ratio between the surface area that provides propulsion and the inert surface area.

Our paper then introduces our solution for this problem, a system called “Integrated Joint Actuator” (IJA) for serpentine mobile robots. The IJA uses specially designed pneumatic bellows as actuators in combination with our unique Proportional Position and Stiffness (PPS) control system.

While the combination of requirements defined in this paper may be unique to serpentine robots, subsets of these requirements are typically found in many existing mechanical structures. Our IJA should thus also appeal to researchers outside of the small community of serpentine robot developers.
In spite of the commonly known advantages of electric actuators we chose a different type of actuator as we identified one overriding advantage of pneumatic actuation: natural compliance. Among pneumatic actuators we chose bellows because of their unique quality of fitting optimally into the shape-changing joint space of serpentine robots. This choice was further supported by additional benefits of bellows: frictionless motion and very large actuation strain.

In addition our paper presents a unique control method for these actuators. Our method, called "proportional position and stiffness control" allows the simultaneous, proportional control of stiffness (compliance) and position of the joints, without wasting compressed air at steady state.

Experimental results presented in this paper illustrate the suitability of our IJA to the unique requirements of serpentine robots. Having completed the development of the fully functional IJA, we intend to focus future research on the global control of serpentine robots with special attention to the coordination of joint during motion.

\section{ACKNOWLEDGMENT}

This work was funded by the U.S. Department of Energy under Award No. DE-FG04-86NE3796. We are also grateful to graduate student Malik Hansen for his work on designing and building the OmniTread segments.

\section{REFERENCES}

[1] AOKI, T.; Ohno, H.; Hirose, S.; "Design of Slim Slime Robot II (SSRII) with Bridle Bellows," Proc. IEEE/RSJ International Conference on Intelligent Robots and System, pp. 835-840, vol.1, Oct. 2002

[2] BOBROW J.E., McDonell B.W., "Modeling, identification, and control of a pneumatically actuated, force controllable robot," IEEE Trans. on Robotics and Automation, vol.14, no.5, pp. 732-741, 1998.

[3] BROCKMANN W., Kohne J.: "Towards high-speed, motion control of pneumatic actuators with low-cost valves," $4^{\text {th }}$ Int. Conference on Climbing and Walking Robots CLAWAR, pp. 339-346, Karlsruhe, Germany 2001.

[4] BROWN, H.B., Jr.; Vande Weghe, J.M.; Bererton, C.A.; Khosla, P.K.; "Millibot trains for enhanced mobility," IEEE/ASME Transactions on Mechatronics, Vol. 7, Issue 4 , pp. 452-461, Dec. 2002

[5] CRAIG J.J., Introduction to robotics, Addison-Wesley Publishing Company, Inc. 1989

[6] DOWLING, K., "Limbless Locomotion: Learning to Crawl with a Snake Robot.” Doctoral Dissertation, tech. report CMU-RI-TR-97-48, Robotics Institute, Carnegie Mellon University, December, 1997. 
[7] GALT, S.; Luk, B.L.; Cooke, D.S.; Collie, A.A., "A tele-operated semiintelligent climbing robot for nuclear applications," Proc. Fourth Annual Conference on Mechatronics and Machine Vision in Practice, pp. 118123, 23-25 Sept. 1997

[8] GRANOSIK G., Jezierski E., "Application of a maximum stiffness rule for pneumatically driven legs of walking robot," Proc. of $2^{\text {nd }}$ Int. Conference on Climbing and Walking Robots, pp.213-218, Portsmouth, UK 1999.

[9] HIROSE, S. and Morishima, A., 1990, "Design and Control of a Mobile Robot With an Articulated Body.” The International Journal of Robotics Research, Vol. 9, No. 2, pp. 99-113, April 1990

[10] HIROSE, S., Morishima, A., Tukagosi S., Tsumaki T., Monobe H., "Design of Practical Snake Vehicle: Articulated Body Mobile Robot KR-II,” Fifth International Conference on Advanced Robotics, 'Robots in Unstructured Environments', pp 833 -838 vol.1, June 1991

[11] HUBER, J. E., Fleck, N. A. and Ashby, M.F. "The selection of mechanical actuators based on performance indices," Proc. of the Royal Society of London. Series A. 453, pp. 2185-2205, UK 1997.

[12] KIMURA, H.; Hirose, S.; "Development of Genbu : Active wheel passive joint articulated mobile robot," Proc. IEEE/RSJ International Conference on Intelligent Robots and System, pp. 823 -828 vol.1, Oct. 2002

[13] KLAASSEN, B. and Paap, K.L., "GMD-SNAKE2: A Snake-Like Robot Driven by Wheels and a Method for Motion Control," Proc. of 1999 IEEE International Conference on Robotics and Automation, pp. 30143019, Detroit, MI, May 10-15, 1999.

[14] LIU R., Zong G., Wang S., Bi S, "On the implementation of direct compliant control on parallel mechanism using rubberactuators," Proc. ASME Japan/USA Symposium on Flexible Automation, pp. 120-123, Boston, MA, 1996

[15] LONG G., Anderson J., and Borenstein J., "The OmniPede: A New Approach to Obstacle Traversion," Proc. of IEEE International Conference on Robotics and Automation, pp. 714-719, USA 2002

[16] MORI, M.; Hirose, S.; "Three-dimensional serpentine motion and lateral rolling by active cord mechanism ACM-R3," IEEE/RSJ International Conference on Intelligent Robots and System, pp. 829-834 vol.1, Oct. 2002

[17] MUTH, J. and Grant, E., 2000, “A Safer Way to Search Disaster Sites,” IEEE Robotics \& Automation Magazine, pp. 56-57, Sept. 2000

[18] OHNO, H.; Hirose, S.; "Study on slime robot (proposal of slime robot and design of slim slime robot)," Proc. IEEE/RSJ International Conference on Intelligent Robots and Systems, (IROS 2000), pp 22182223, Vol 3, Nov. 2000

[19] OLSZEWSKI M., Janiszowski K., "Solving of the main problems of position control of pneumatic drives of machinery and industrial robots," (in polish) Proc. of $2^{\text {nd }}$ Conference on Mechatronics, pp.69-74, Warsaw, 22-23 Sept. 1994

[20] OSUKA K, and Kitajima, H., "Development of Mobile Inspection Robot for Rescue Activities: MOIRA,” Proc. of the 2003 IEEE/RSJ Intl. Conference on Intelligent Robots and Systems, Las Vegas, Nevada, October 2003.

[21] ROBINSON D.W., "Design and analysis of series elasticity in closedloop actuator force control,” PhD thesis, Massachusetts Institute of Technology 2000

[22] SHEARER J.L., "Study of pneumatic processes in the continuous control of motion with compressed air I, II," Trans. ASME, pp. 233-249, Feb. 1956

[23] SCHOLL, K.-U.; Kepplin, V.; Berns, K.; Dillmann, R.; "Controlling a multi-joint robot for autonomous sewer inspection," Proc. IEEE International Conference on Robotics and Automation, pp. 1701 -1706 vol.2, April 2000

[24] SHIH M.C., Ma M.A., "Position control of a pneumatic rodless cylinder using sliding mode M-D-PWM control the high speed solenoid valves," JSME International Journal, Series C, vol.41, no.2, pp. 236-241, 1998

[25] TAKAYAMA, T.; Hirose, S.; "Development of Souryu-I connected crawler vehicle for inspection of narrow and winding space," 26th Annual Conference of the IEEE Industrial Electronics Society, IECON 2000, pp. 143 -148 vol.1, Oct. 2000

[26] VAN VARSEVELD R.B., Bone G.M, "Accurate position control of a pneumatic actuator using on/off solenoid valves," IEEE/ASME Transactions on Mechatronics, vol.2, no.3, pp. 195-204, 1997.

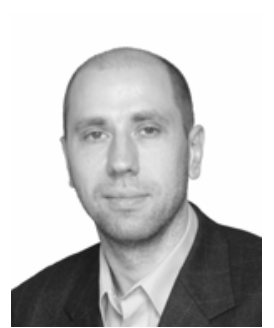

G. Granosik was born in Poland in 1970. He received the M.Sc. and D.Sc. in Robotics and Automation in 1994 and 2000, respectively, from Technical University of Lodz, Poland. Since 1994 he has been working at the Technical University of Lodz as Assistant, from 2000 till 2002 as Adjunct, and since 2005 as Lecturer. From 2002 till 2004, Dr. Granosik worked as postdoctoral researcher in the Mobile Robotics Laboratory at the University of Michigan, Ann Arbor. He is author and co-author of more then 25 publications. His research interests include designing of novel robots, robot control systems and pneumatic drives.

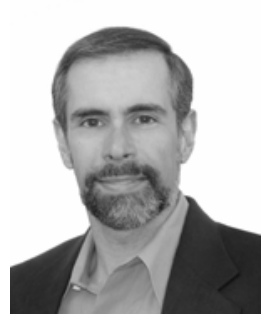

J. Borenstein received the B.Sc., M.Sc., and D.Sc. in mechanical engineering in 1981, 1983, and 1987, respectively, from the Technion - Israel Institute of Technology. He currently holds the rank of Research Professor at the University of Michigan, where he headed the Mobile Robotics Laboratory since 1987. His research interests include mobile-robot position estimation, obstacle avoidance, and the design of novel robotic platforms. He has over 120 publications on mobile robotics and won the 1998 Discover Magazine Award for Technological Innovation for his invention of the GuideCane. 